\title{
LA INTERSECCIONALIDAD DEL TRABAJO. LAS TRANSFORMACIONES EN EL TRABAJO DE LAS MUJERES EN ARGENTINA
}

\section{THE INTERSECTIONALITY OF WORK. THE TRANSFORMATIONS IN THE FEMININE WORK IN ARGENTINA}

\author{
Marianela Scocco
}

Fecha de recepción: 7 de setiembre de 2017 - Fecha de aceptación: 18 de diciembre de 2017

\begin{abstract}
Resumen
Este artículo indaga sobre la incorporación masiva del trabajo de las mujeres a una nueva división social y sexual del trabajo en el marco de una crisis internacional del modelo de acumulación capitalista en los años setenta. Se analiza la categoría de género atravesada por las de trabajo y clase. Complementariamente, se precisan algunos apuntes sobre cómo se efectuaron dichas transformaciones en el mundo del trabajo en la Argentina a partir de la década de 1980, para visualizar el problema en un caso concreto. Por último, se utiliza la expresión clase-que-vive-del-trabajo del sociólogo brasilero Ricardo Antunes para otorgar validez contemporánea al concepto marxista de clase trabajadora y, de esta forma, poner en discusión las relaciones entre clase y género. Concluimos que, en un proceso más profundo de emancipación del género humano, es necesaria la acción conjunta e indispensable entre hombres y mujeres que pertenezcan a la clase trabajadora. Pero la lucha de las mujeres por su emancipación es también una acción contra las formas socio-históricas de la opresión masculina. Y en este sentido, la lucha feminista es precapitalista y será además poscapitalista, ya que el fin de la sociedad de clases no significa directamente el fin de la opresión de género.

Palabras clave: Capital, trabajo, clase, género, mujer.
\end{abstract}

\begin{abstract}
This article investigates the massive incorporation of female labor into a new social and sexual division of labor in the context of an international crisis of the model of capitalist accumulation in the 1970s. It analyzes the category of gender traversed by those of work and class. Complementarily, some notes are needed on how these transformations were made in the world of work in Argentina from the 1980s, to visualize the problem in a concrete case. Finally, the expression life-work-class of the Brazilian sociologist Ricardo Antunes is used to give contemporary validity to the Marxist concept of the working class and, in this way, to discuss the relations between class and gender. We conclude that, in a deeper process of emancipation of the human race, there is a need for joint and indispensable action among men and women who belong to the working class. But the struggle of women for their emancipation is also an action against the socio-historical forms of male oppression. And in this sense, the feminist struggle is precapitalist and will also be post-capitalist, since the end of class society does not directly signify the end of gender oppression.
\end{abstract}

Keywords: Capital, work, class, gender, woman

1 Universidad Nacional de Rosario (UNR)-CONICET, Argentina, maria_nob4@hotmail.com 


\section{Introducción}

En los años setenta, el llamado toyotismo y la era de la acumulación flexible emergieron en Occidente. Por esa época comenzaba la crisis capitalista -que perdura hasta hoy-, expresada como crisis del modelo de acumulación taylorista-fordista. Esto provocó que el capital implementase una enorme transformación. Estas reestructuraciones, originadas en la propia competencia intercapitalista, surgieron también de la necesidad de controlar las luchas sociales nacidas del mundo del trabajo. Por eso, el capital inició un proceso de reorganización de sus formas de dominación de la sociedad. Estas mutaciones, posibilitaron la aparición de un/a trabajador/ra polivalente y multifuncional, cuya finalidad es, en última instancia, la intensificación de las condiciones de explotación de la fuerza de trabajo, reduciendo y hasta eliminando el trabajo improductivo, es decir, el trabajo que no crea valor, como las actividades de mantenimiento, acompañamiento e inspección de calidad, que pasaron a ser directamente incorporadas al trabajo productivo (Antunes, 2005). Estas transformaciones tuvieron resultados muy negativos en el mundo del trabajo: desregulación de los derechos del trabajo, es decir, lo que argentinos y argentinas conocemos como flexibilización laboral; precarización y tercerización de la fuerza de trabajo; fragmentación en la clase trabajadora, con la conversión del sindicalismo de clase en un sindicalismo dócil y disciplinado, de asociación, meramente un "sindicalismo de empresa"; y, finalmente, la incorporación masiva del trabajo de las mujeres a una nueva división social y sexual del trabajo, imponiendo sobre la labor de la mujer una mayor intensidad de precariedad y explotación, así como también el crecimiento de la cantidad de trabajadores y trabajadoras inmigrantes que ejecutan trabajos sin calificación y frecuentemente ilegales.

Esto se estructura preservando dentro de las fábricas centrales un número reducido de trabajadores y trabajadoras medianamente calificados/as, multivalentes y, al menos en apariencia, comprometidos/das con su empresa, así como ampliando el conjunto fluctuante de trabajadores y trabajadoras temporarios/as y precarizados/as.

Este artículo examina la incorporación masiva del trabajo de las mujeres a una nueva división social y sexual del trabajo en el marco de esa crisis internacional del modelo de acumulación capitalista en los años setenta. Complementariamente, se precisan algunos apuntes sobre cómo se efectuaron dichas transformaciones en el mundo del trabajo en la Argentina a partir de la década de 1980, para visualizar el problema en un caso concreto. Por último, se utiliza la expresión clase-que-vive-del-trabajo del sociólogo brasilero Ricardo Antunes para otorgar validez contemporánea al concepto marxista de clase trabajadora y, de esta forma, poner en discusión las relaciones entre clase y género.

\section{Las intersecciones entre género y clase}

El pensamiento posmoderno, por recusar la idea de totalidad, postula que hay que analizar las categorías de género, generación y origen sin atravesarlas con las de trabajo o clase porque éstas últimas, según esta perspectiva, dejaron de existir. Sin embargo, la esfera del trabajo nos permite ver las transversalidades existentes entre estas tres dimensiones del género humano. Para las perspectivas de género, la división sexual del trabajo, como afirma María Antonia Carbonero Gamundi (2007), está atravesada por las relaciones de género que regulan la manifestación simbólica de la responsabilidad de lo masculino o femenino y que se producen y reproducen en la interacción cotidiana. Estos patrones que disciplinan el comportamiento constituyen lo que denominan "modelos culturales de género" y, según esta perspectiva, entre ellos juegan un lugar central los que inciden en la división sexual del trabajo y en particular del trabajo en el hogar y la maternidad. Pero no se plantean la división sexual del trabajo atravesada por las distintas clases sociales y los diferentes momentos históricos. Como afirma Helena Hirata (2000), las relaciones de clase o relaciones de sexo, los antagonismos de clases o antagonismo de sexo, se interpretan a menudo como si la importancia dada a una de esas relaciones implicaba dejar la otra en un plano secundario. Daniel Kergoat (citado en Hirata, 2000) conceptualizó esas dos relaciones 
sociales en términos de "co-extensividad", es decir en términos de superposiciones parciales entre uno y otro. Así lo analiza Hirata:

Si partimos de esta idea de co-extensividad, la explotación en el trabajo asalariado y la opresión de sexo son indisociables, las esferas de la explotación económica -o de las relaciones de clases- son simultáneamente aquellas donde se ejerce el poder machista sobre las mujeres (Hirata, 2000, p. 2).

Las opresiones de género, clase y origen han sido un tema recurrente en la literatura feminista, con la emergencia de la perspectiva interseccional de los feminismos postcoloniales. Interseccionalidad se refiere a la interacción entre el género, clase, origen y otras categorías de diferenciación en la vida de las personas en las prácticas sociales, en las instituciones e ideologías culturales. Diversas autoras han explorado la complejidad teórica de la interseccionalidad. Tomamos el concepto de Castellanos y Baucells (2017) que se ocupan de la cuestión de la exclusión, que hace visible el posicionamiento múltiple que constituye la vida cotidiana y las relaciones de poder. Como sostienen las autoras:

La interseccionalidad posibilita ver las particularidades de la diferenciación y las divisiones sociales. Las fronteras son simbólicas, pero se experimentan de manera real y se visibilizan en el rechazo/aceptación de la diferencia: cuando se es mujer, inmigrante, e indígena y demás dimensiones contenidas en el individuo (Castellanos y Baucells, 2017).

Llegados a este punto, debemos problematizar acerca de por qué se han separado usualmente los conceptos de género y clase social. Por un lado, el "feminismo occidental-blanco-heteropatriarcal", borró las categorías de clase y origen, entre otras, para centrarse en la defensa de la identidad femenina (Castellanos y Baucells, 2017). Esto dejó un legado de exclusiones y entonces el sujeto "mujeres" prescinde de las indígenas, musulmanas, negras, lesbianas e inmigrantes. No obstante, por otro lado, desde los años '70 la izquierda se ha sentido traicionada por el feminismo, se dio cuenta de que esta perspectiva conllevaba implicaciones que van más allá de la "cuestión de la mujer" y que representa una ruptura con su política pasada y presente, tanto respecto a las mujeres como al resto de la clase obrera. Como sostenía Cox (1975):

el sectarismo que la izquierda ha demostrado tradicionalmente en relación con las luchas feministas es una consecuencia de su interpretación reduccionista del alcance y de los mecanismos necesarios para el funcionamiento del capitalismo así como de la dirección que la lucha de clases debe tomar para romper este dominio.

En nombre de la "lucha de clases", la izquierda siempre ha seleccionado a determinados sectores de la clase obrera como sujetos revolucionarios y ha condenado a otros a un rol meramente solidario en las luchas que estos sectores llevaban a cabo, entre ellos a las mujeres.

De esta forma, desde el feminismo posmoderno y desde la izquierda, se ha dejado de lado la interseccionalidad entre clase social y género, hecho que analizamos desde la incorporación del trabajo de las mujeres a una nueva división social y sexual del trabajo tras la crisis internacional del modelo de acumulación capitalista en los años setenta.

\section{La incorporación del trabajo de las mujeres a una nueva división social y sexual del trabajo}

En el mundo contemporáneo del trabajo se experimenta un aumento significativo del componente de las mujeres -que llega a ser de más del $40 \%$ en países avanzados- relacionado, como dijimos, con el trabajo part time, temporario, precarizado y desregulado. Esto tiene un signo inverso en la desigualdad salarial de las mujeres -en Brasil es del 70\% menor- y también existen divergencias en relación a los derechos laborales y a las condiciones de trabajo. En la división sexual del trabajo, aplicada por el capital dentro de la fábrica, generalmente las actividades de concepción o las basadas en capital 
intensivo son llevadas a cabo predominantemente por varones, mientras que las actividades de menos calificación, más simples y artesanales y fundadas en trabajo intensivo son dedicadas a las mujeres.

No obstante, la ampliación del trabajo de las mujeres a partir de los años setenta es, en parte, el resultado de una decisiva lucha femenina por el incremento de la participación de la mujer en el mercado de trabajo y, en este sentido, es una lucha emancipatoria para las mujeres porque es un paso para disminuir la dominación patriarcal del hogar. Pero el capital, al incorporar a las mujeres al mercado de trabajo lo hace desigualmente, y además, abrió considerablemente el espacio de trabajo de las mujeres a jornadas de tiempo parcial para que las mujeres hagan en su casa la segunda jornada de trabajo, que tiene como finalidad, entre otras cosas, la reproducción de la clase trabajadora, componente imprescindible para la reproducción del capital. Entonces, si queremos mejorar las condiciones laborales de las mujeres y los varones, así como sus condiciones de vida, es fundamental entender que la reproducción social es una responsabilidad de todos, y no solamente de las mujeres.

En el mismo sentido, el capital ha sabido también apropiarse de la polivalencia y multiactividad de las mujeres. En la medida en que los varones - por condiciones socio-históricas- muestran más dificultad para adaptarse a nuevas situaciones polivalentes, que comprenden niveles más profundos de explotación, el capital ha utilizado este atributo social heredado por las mujeres.

Por último, los sindicatos frecuentemente excluyen a las mujeres trabajadoras de sus filas, además de mostrarse incapaces de incluir a trabajadores y trabajadoras tercerizados/as y precarizados/as en general. Sucede, como veremos más adelante, que hoy la clase trabajadora está compuesta crecientemente por estos segmentos diferenciados.

Pero además las transformaciones que ocurrieron bajo el proceso de reorganización de las formas de dominación de la sociedad del capitalismo y sus resultados en el mundo del trabajo, de los cuales uno es la incorporación masiva del trabajo de las mujeres a una nueva división social y sexual del trabajo, impusieron sobre el trabajo de la mujer una mayor intensidad de precariedad y explotación.

\section{Las transformaciones del modelo de acumulación capitalista en la Argentina}

En adelante, detallaremos algunos apuntes sobre cómo se efectuaron dichas transformaciones en la Argentina a partir de los años ochenta, para visualizar el problema en un caso concreto. Aún así, estas mutaciones se efectuaron en la mayor parte del mundo occidental capitalista.

En Argentina, previamente, durante el período de expansión industrial iniciado en los años treinta y profundizado en los cuarenta, el empleo de las mujeres mostró un incremento permanente y diferenciado por niveles educativos y pertenencia de clase. Las actividades laborales de las mujeres, hacia las décadas del cuarenta y cincuenta, se concentraban en el sector industrial -sobre todo en la rama textil-y en el terciario, expresándose así un desarrollo de ciertas actividades consideradas "femeninas".

En las décadas del sesenta y setenta, a partir de la aplicación de una estrategia de carácter desarrollista, orientada a una industrialización basada en la incorporación de tecnologías y expansión de empresas de capital intensivo, la demanda de mano de obra cambió, en busca de mayor calificación, al mismo tiempo que el sector terciario creció. Este proceso, que afectó a mujeres y varones, demandó personal con mayor nivel educativo que, en el caso del trabajo de las mujeres, engrosó las filas tanto del sector industrial como del terciario.

Recién en los años ochenta comenzó a percibirse los efectos del proceso de desindustrialización iniciado durante la última dictadura militar (1976-1983) en Argentina ${ }^{2}$. Este nuevo contexto económico produjo modificaciones sustanciales en las características de inserción de la mujer al mercado

2 El régimen iniciado con la última dictadura militar logró imponer, a través del terror y la violencia, un nuevo modelo de acumulación basado en la primacía del capital financiero, desplazando a la producción de bienes industriales, y modificaron notoriamente la configuración orgánica y subjetiva de las fuerzas políticas y sociales anteriores al golpe de 1976. 
de trabajo. Hasta mediados de los años setenta se podía interpretar que la participación femenina en el mundo del trabajo respondía fundamentalmente a un proceso de modernización que operaba como motor de transformación cultural y social, sobre todo en ciertos sectores de mayor nivel educativo y poder adquisitivo. En cambio, a raíz de la profunda reestructuración económica que se inició en 1976, el aumento del trabajo de las mujeres pareció vincularse más con la figura del trabajador complementario que se incorporó al mercado para compensar el deterioro de los ingresos del jefe de hogar y su relación laboral estuvo signada por condiciones de precariedad, alta participación en los índices de desocupación y menor nivel salarial que los varones en actividades similares.

La década del ochenta en la Argentina, y en general en Latinoamérica, muestra entonces un mercado de trabajo en permanente deterioro, con tasas de desocupación en aumento y distintas formas de precarización de la fuerza de trabajo como reflejo de una economía estancada en su producto industrial, carente de inversiones y con un agudo proceso inflacionario que fue mellando por el poder adquisitivo de la clase trabajadora (Levin; Campostrini; Sosa y Voras, 2007).

La crisis que se ha instalado en Argentina desde los años ochenta ha tenido, y sigue teniendo, consecuencias profundas sobre la familia y los modos de vida de sus miembros. Mientras la desocupación ha llegado a niveles no igualados antes en la historia del país, atacando ahora no sólo a varones jóvenes, sino especialmente a los jefes de hogar, un número creciente de mujeres casadas y unidas, madres y cónyuges de edades medias, ha salido a trabajar para aportar ingresos a los deteriorados presupuestos familiares. Después, las mujeres no se retiraron; es decir, no siguieron siendo mano de obra complementaria sino que permanecieron en el mercado laboral.

Como sostiene Catalina Wainerman:

\begin{abstract}
Este movimiento de más mujeres fuera del hogar no es ahora, como se interpretaba en los años setenta, sólo evidencia de la modernización de la sociedad ni de la ampliación de oportunidades que motorizan una reversión de la condición secularmente postergada de las mujeres. En un país empobrecido, gran parte de las mujeres que trabajan o buscan trabajo han salido a reemplazar los ingresos deteriorados de sus cónyuges buscando frenar la caída ‘cuenta abajo' de sus familias (Wainerman, 2007, pp. 147-148).
\end{abstract}

Otras mujeres de sectores más favorecidos han alcanzado niveles más altos de educación. En ambos casos, las mujeres de distintas clases sociales tienden a incorporarse masivamente a la actividad asalariada y a recorrer trayectorias laborales cada vez más duraderas y menos interrumpidas por circunstancias familiares. Para Catalina Wainerman (2007), esta nueva situación es totalmente revolucionaria. Sin embargo, como ha sostenido Alma Espino (2010) en su estudio sobre Latinoamérica, las tasas más bajas de participación laboral de las mujeres se dan en los sectores más pobres. Las mujeres en condiciones de pobreza tienen una cantidad de restricciones de diverso orden para decidirse a participar en el mercado laboral: cultural, material, número de hijos, de segregación residencial de las ciudades. Un rasgo característico del tipo de inserción laboral de las mujeres es la restricción del tiempo disponible para dedicarse al mercado laboral, a causa de la carga de trabajos no remunerados que asumen, por la asignación cultural y social que está lejos de ser superada (Espino, 2010).

Por último, otros factores que estimularon la inserción de las mujeres en el mercado laboral fueron la reestructuración productiva y la emergencia de las industrias de exportación. Este proceso tuvo lugar en los países como Argentina donde las reformas estimularon la producción manufacturera de algunos productos primarios y sus exportaciones, al mismo tiempo que la apertura significó básicamente una apertura importadora de productos industriales. En estos casos, las mujeres tendieron a emplearse en el sector de servicios, desde los sistemas financieros donde el nivel de empleo es bastante alto, como en el otro extremo, trabajando en empresas tercerizadas que aseguran la limpieza, la seguridad y otros servicios que se traducen en menor calidad en términos de salarios y condiciones de trabajo, como los call center por ejemplo (Espino, 2010). 
Así, siguiendo a Catalina Wainerman, a partir de los años ochenta, en Argentina pero también en el resto del mundo capitalista, “(...) el crecimiento de la participación económica de las mujeres no aparece vinculado al mejoramiento de su situación en la sociedad, sino a procesos de ajuste estructural y reestructuración económica" (Wainerman, 2007, p. 157). Por tanto, para que este progreso en la calidad de vida de las mujeres se realice efectivamente, es necesario superar primero las condiciones sociales de opresión que instala el sistema capitalista, y para ello, será indispensable plantearse un plan de lucha que contemple tanto las perspectivas de género como las de clase.

\section{Relaciones entre género y clase}

Los elementos analizados en el apartado anterior permiten avanzar en las difíciles pero decididamente necesarias relaciones entre género y clase.

La lucha de clases es también femenina. Podrán propiciar condiciones de igualdad en tanto el género se realice en una sociedad sin clases sociales. ${ }^{3}$ La emancipación parcial femenina para ser una emancipación total debe ser también una emancipación de clase, debe articular género y clase y género y trabajo. Esto es lo que omite constantemente la visión de género posmoderna y la izquierda tradicional. Por eso aquí es pertinente hacer una aclaración en relación a la vigencia de la categoría marxista de clase. El sociólogo brasilero Ricardo Antunes utiliza la expresión clase-que-vive-del-trabajo, según su afirmación, para otorgar validez contemporánea al concepto marxista de clase trabajadora. Siguiendo a Antunes:

La clase-que-vive-del-trabajo, la clase trabajadora hoy, incluye a todos aquellos que venden su fuerza de trabajo, teniendo como núcleo central a los trabajadores productivos (...) Siendo el trabajador productivo aquel que produce directamente plusvalía y que participa directamente del proceso de valorización del capital (Antunes, 2005, pp. 91-92).

Pero esta clase engloba también a trabajadores y trabajadoras improductivos, “(...) aquellos cuya forma de trabajo es utilizada como servicio, ya sea para uso público o para el capitalista, y que no se constituyen como elemento directamente productivo" (Antunes, 2005, p. 92). El trabajo improductivo abarca un amplio abanico de asalariados y asalariadas: desde los y las insertos en el sector de servicios, bancos, comercio, servicios públicos, turismo, etcétera; hasta los y las que realizan actividades en las fábricas pero no crean valor en forma directa. Por tanto, la noción de la clase-que-vive-del-trabajo incluye a la totalidad de los trabajadores y trabajadoras asalariados/as, es decir, a todos/as aquellos/ as que venden su fuerza de trabajo a cambio de un salario. Sin embargo, esta clase excluye a quienes Antunes denomina gestores del capital, que son aquellos/as que detentan la función del control en el proceso de trabajo, de valorización y reproducción del capital en el interior de las empresas y que reciben ingresos elevados.

\section{Conclusión}

Como decíamos más arriba, la mujer trabajadora realiza generalmente una doble jornada laboral, dentro y fuera de su casa. Al hacerlo, además de la duplicación de la actividad laboral, ella es doblemente explotada por el capital: ejerce, en el espacio público, su trabajo productivo en el ámbito laboral y, en su vida privada, consume horas en el trabajo doméstico, donde genera las condiciones indispensables para la reproducción de la fuerza de trabajo (Federici, 2013). Por eso, siguiendo a Helena Hirata, cuando

3 La abolición de las clases sociales es uno de los hitos principales que diferentes revolucionarios y teóricos del marxismo han promovido para la construcción del socialismo, entendido a éste como un período de transición entre el capitalismo y el comunismo. Entre ellos del propio Karl Marx, Lenin, Mao Zedong y el Ernesto "Che” Guevara. Es condición fundamental para el comunismo la construcción de una sociedad sin clases. (Scocco, 2018). 
se problematiza acerca del trabajo de las mujeres, se debe incorporar también al trabajo no remunerado, es decir, no asalariado.

Por tanto, como concluye Antunes: “(...) el capital convierte lo que en un momento real -aunque todavía limitado- de emancipación parcial de las mujeres frente a la explotación del capital y la opresión masculina, en una fuente de más intensa desigualdad" (Antunes, 2005, p. 100).

Todo esto se complejiza cuando aparece la interseccionalidad, articulando además de la categoría de género, las ya mencionadas de generación y origen. Porque, en algún sentido, una mejor blanca tiene menos precarización laboral que un hombre negro o, en el corte generacional, hombres y mujeres de más de cuarenta años están fuera del mercado de trabajo. Cuando articulamos esto con la categoría de clase, la complejidad es mayor, porque hay hombres y mujeres burgueses negros que subordinan a hombres y mujeres blancas de la clase trabajadora. Es decir, como sostienen Castellanos y Baucells (2017): "cuando las mujeres negras comparten subordinaciones con hombres negros y mujeres blancas y, al mismo tiempo, son diferentes a los hombres negros y mujeres blancas."

Por todo ello, es necesario un proceso más profundo de emancipación del género humano, con una acción conjunta e indispensable entre hombres y mujeres que pertenezcan a la clase trabajadora. Pero la lucha de las mujeres por su emancipación es también una acción contra las formas socio-históricas de la opresión masculina. Y en este sentido, la lucha feminista es precapitalista y será además poscapitalista, ya que el fin de la sociedad de clases no significa directamente el fin de la opresión de género. Aunque el fin de la sociedad de clases supone generar una forma social libre, autodeterminada y emancipada, que posibilitará la aparición de condiciones sociales capaces de ofrecer bases de igualdad entre hombres y mujeres. Sin embargo, si bien el primer objetivo, alcanzar una sociedad sin clases, es tarea de uno y otro; el segundo desafío, la emancipación específica de la mujer con relación a la opresión masculina, es una empresa prioritariamente de las mujeres, a la que los varones pueden y deben sumarse pero sin la función de mando y control.

\section{Referencias}

Antunes, Ricardo (2005) Los sentidos del Trabajo. Ensayo sobre la afirmación y la negación del trabajo, Buenos Aires: Herramienta Ediciones.

Carbonero Gamundi, María Antonia (2007) Intersecciones de género, clase y poder: políticas y prácticas de cuidado en la Unión Europea. En Carbonero Gamundi; María Antonia y Levín, Silvia (comp.), Entre Familia y Trabajo. Relaciones, conflictos y políticas de género en Europa y América Latina, Rosario: Homo Sapiens Ediciones.

Castellanos, Rosa y Baucells, Olga (2017) "Interseccionalidad del género y mercado de trabajo postfordista". La ventana vol.5 no.46 Guadalajara jul./dic. 2017. Recuperado de http://www.scielo.org.

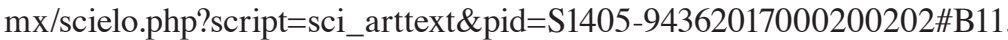

Cox, Nicole (1975) "Contraatacando desde la cocina”. En Federici Silvia (2013) Revolución en punto cero. Trabajo doméstico, reproducción y luchas feministas, Madrid: Traficantes de Sueños.

Espino, Alma (2010) "Género y mercados laborales en América Latina”. En AA.VV, La globalización al debate. Reflexiones feministas, Lima: Cladem.

Federici Silvia (2013) Revolución en punto cero. Trabajo doméstico, reproducción y luchas feministas, Madrid: Traficantes de Sueños.

Hirata, Helena (2000) "Relaciones sociales de sexo y división del trabajo. Contribución a la discusión sobre el concepto trabajo", Herramienta, $\mathrm{N}^{\circ}$ 14. Recuperado de http://www.herramienta.com.ar.

Levin, Silvia; Campostrini, Adela; Sosa, Ruth y Voras, Claudia (2007) "La política de género de las políticas públicas en Argentina”. En Carbonero Gamundi; María Antonia y Levín, Silvia (comp.), Entre Familia y Trabajo. Relaciones, conflictos y políticas de género en Europa y América Lati$n a$, Rosario: Homo Sapiens Ediciones. 
Scocco Marianela (2018) "La transición del capitalismo al comunismo", Izquierdas 42, octubre 2018: 186-197. Recuperado de: http://izquierdas.cl/images/html/n42/index42.html

Wainerman, Catalina (2007) "Familia, trabajo y relaciones de género". En Carbonero Gamundi; María Antonia y Levín, Silvia (comp.), Entre Familia y Trabajo. Relaciones, conflictos y políticas de género en Europa y América Latina, Rosario: Homo Sapiens Ediciones. 